\title{
Selection of the optimum device parameters for constant magnetic field generation
}

\author{
Regina Khazieva ${ }^{1, *}$, and Maksim Ivanov $^{1}$ \\ ${ }^{1}$ Ufa State Petroleum Technological University, 450044, Kosmonavtov street, 1, Ufa, Russia
}

\begin{abstract}
The aim of the work is to develop a device design for creating constant magnetic fields and select its parameters, select an electrical circuit for powering the device and determine how the energy efficiency of the device changes with an increase in its overall dimensions. When solving the problem, the KOMPAS-3D three-dimensional modeling system was used, the magnetic field induction was calculated using the Pascal ABC programming system, the optimal device parameters were selected using the Microsoft Excel program. Calculations have shown that the coefficient taking into account the inhomogeneous distribution of the magnetic field in the device is 0.883 . The optimum ratio between the height and the radius of the cylinder, along which the emulsion flows, is $h=r \sqrt{20}$. Using the KOMPAS3D three-dimensional modeling system, a model of the device under development was built taking into account the obtained relationships. When using a circuit with an uncontrolled rectifier and autotransformer, the power consumed by the device is 2.67 times lower than the power consumed by the device when using a circuit with a controlled rectifier and a transformer. Energy efficiency increases significantly with the increase in device performance.
\end{abstract}

\section{Introduction and literature review}

Electromagnetic treatment of water systems can be used to achieve results in various industries: for the production of concrete, reducing the formation of scale [1], flotation beneficiation of minerals, thickening and filtration of suspensions, as well as for many other purposes. With the help of magnetic fields, sea water is treated [2], water is purified from impurities [3], various media are heated [4]. In the oil and gas industry, this method can be used for dehydration and desalination of oil-water emulsions [5-7]. The destruction of oil-water emulsions in a magnetic field is based on its effect on the armor shells of oil globules in water, which consists in loosening the armor shells at the oil-water interface, which occurs due to the movement of iron compounds towards the sources of the magnetic field [8]. In emulsions of the opposite type, "water in oil", already water droplets will stretch along the lines of force, experiencing the pressure of the surrounding oil, while the thickness of the armor layer will decrease, which contributes to the dehydration process [9]. The use of constant magnetic fields to intensify the process of oil preparation is also mentioned in publications $[10,11]$. Also, there is a method for separating an oil-water emulsion, which consists in exposure to an electric field [12-14], but its implementation requires high values of the applied voltage.

Works devoted to the use of constant magnetic fields for the treatment of water-oil emulsions began to be published relatively recently: on July 20, 2000 Veles
P.R., Pivovarova N.A., and others developed a flow-type magnetizing device, the effect of which on the demulsifier leads to accelerated coalescence of drops, and, as a consequence, dehydrogenation of the emulsion [15]. On May 27, 2001, the same authors proposed a magnetic tunnel that provides a high utilization rate of the magnetic energy of sources and an increase in the reliability of the magnetiser [16]. Subsequently, this system was improved due to a more uniform distribution of the magnetic field over the entire section of the apparatus [17]. According to the studies carried out in the publication [18], magnetic treatment is most effective for more watered oil samples, which emphasizes the relevance of developing a device for magnetic treatment, since oil water cut increases with the operation of field wells. The article [19] shows that under the combined effect of a magnetic field and demulsifiers, the magnetic field enhances the effect of the reagent, and at the maximum value of magnetic induction leads to complete dehydration of oil, which proves the effectiveness of the combined effect of various methods of processing oilwater emulsions. The influence of the geometry of the device on the effectiveness of the action of electromagnetic fields was also studied in the publication [20]. It is necessary to supply a current of a given value to the turns of the coil for create a magnetic induction of a given value.

The current generator was studied in the publication [21]. The power supply of the device under development should be carried out from a secondary power supply source through an inductive-capacitive voltage

\footnotetext{
* Corresponding author: khazievart@mail.ru
} 
converter, the properties of which were studied in publications [22, 23]. A similar calculation of the parameters of the electromagnetic system was carried out in the publication [24], however, a different installation is considered there according to the principle of operation.

This article discusses the parameters of a device for processing an oil-water emulsion in a constant magnetic field. This is the first step in the dehydration process. Then, according to the description of the technological process, treatment is carried out in a pulsed electromagnetic field, which helps to accelerate the process of coalescence of water droplets, and, as a consequence, to increase the efficiency of the dehydration process [25].

The purpose of this work is to determine the optimal parameters of the device being developed, which would provide the highest energy efficiency of the process, as well as the choice of a control scheme for this process. The technical task of the device proposed by the authors of this article is to increase the efficiency of the device for the successful implementation of deep dehydration of an oil-water emulsion. At the moment, this device is at the stage of active development, a patent application is being prepared, and an experimental installation is being assembled. The task is achieved by the fact that in this method of dehydration of an oil-water emulsion, a complex application of a constant magnetic field and high-frequency electromagnetic fields is realized. The emulsion pretreatment is performed in a constant magnetic field, as a result of which the water droplets in the oil stretch along the magnetic lines, since the oil components have a much stronger negative magnetic susceptibility than water. A change in the geometric shape of water globules leads to an increase in their surface area, and, consequently, to a decrease in the thickness of the armor layer, which further facilitates demulsification.

\section{Materials and methods}

The object of the research is a mathematical model of the magnetic field distribution in the proposed device. Its modeling was carried out using the PascalABC programming language. Based on the simulation results, a coefficient was calculated that takes into account the inhomogeneity of the magnetic field distribution inside the device. Then, on the basis of the laws of Bio-SavartLaplace, Ohm and Joule-Lenz, an analytical dependence of the power consumed by the device on its design parameters was obtained. This dependence made it possible to obtain the optimal ratio between the height and radius of the working cylinder, as well as to reveal the optimal diameter of the turns of the coil wound on the cylinder. Then the options for the electrical power supply circuit of the device were studied and the optimal one was selected using analysis and calculations carried out.

By a similar method, the dependence of the power consumed by the device on its overall dimensions was obtained.

\section{Results}

A device for processing oil in a constant magnetic field is shown in Fig. 1:

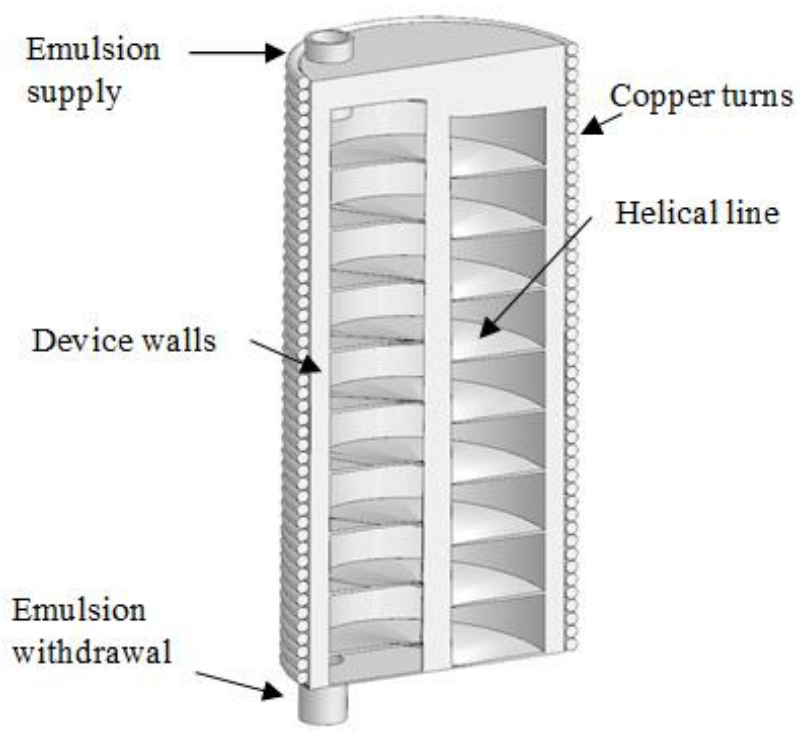

Fig. 1. Device for processing oil in a constant magnetic field.

The device is a vertical cylindrical apparatus consisting of a shell, upper and lower bottoms made of soft magnetic iron. Coils are wound on the shell, with the flow of current through which a constant magnetic field arises inside the apparatus.

The emulsion is fed to the top of the cylinder. The inner walls are equipped with a spiral made of a dielectric material, which does not affect the distribution of the magnetic field inside the device, but ensures the movement of the emulsion practically perpendicular to the lines of force of the magnetic field under the action of gravity. With the help of the angle of inclination of the helix, you can adjust the speed of passage of the emulsion of this device and determine the optimal residence time in a constant magnetic field. At the same time, when water droplets cross the lines of magnetic induction, a force acts on the mechanical impurities located in these droplets, which leads to "loosening" of the armor shells of oil globules in the case of an oil-inwater emulsion, and stretching of water droplets in the case of an emulsion of the type water-in-oil.

According to the dissertation data of A.A. Voltsov "Intensification of the process of stratification of wateroil emulsions by means of their magnetic-vibration treatment" [8], the optimal root-mean-square value of induction when processing in a constant magnetic field is 0.03-0.05 T. We have chosen for further calculations the value of the magnetic induction $0.05 \mathrm{~T}$. Then the magnetic field strength will be equal to $40 \mathrm{kA} / \mathrm{m}$.

We have determined the relationship between the rms value of the magnetic field strength and the current in the turns of the winding of the device.

The article authors carried out a series of calculations based on the Biot-Savart-Laplace law (1) to plot graphs of the dependence of the distribution of magnetic induction in such a system on the coordinate of the point. 


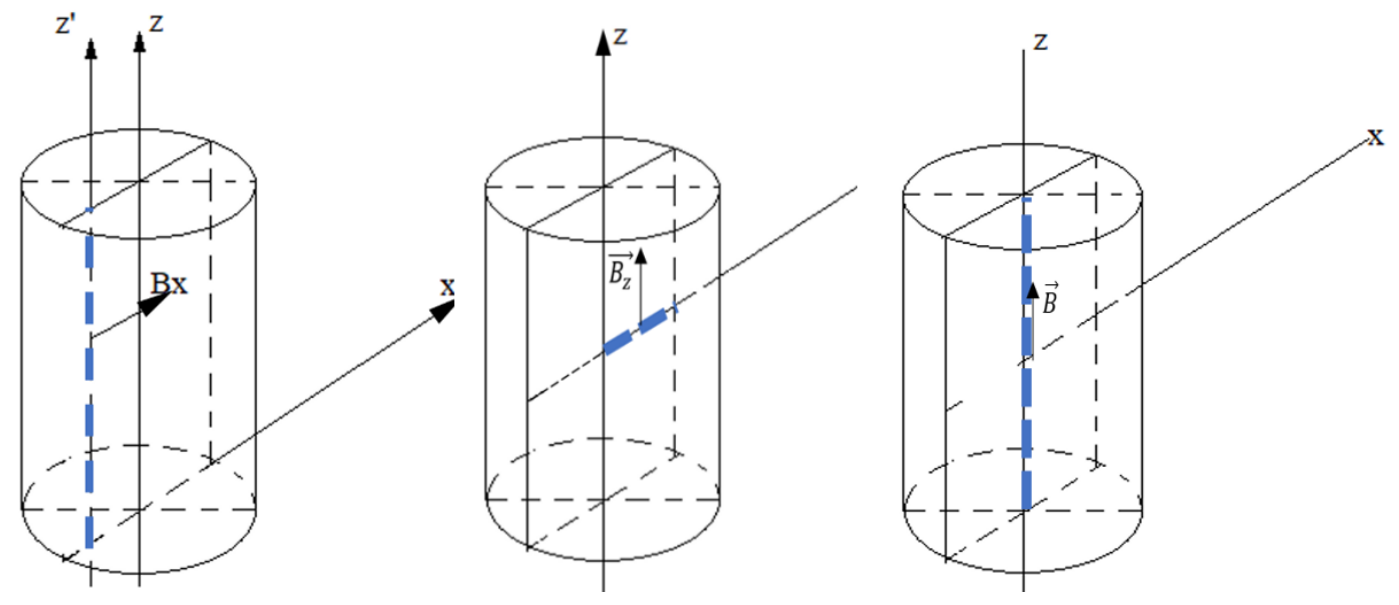

Fig. 2. Contours along which the components of the magnetic induction were calculated: a) circuit for calculating the transverse component of magnetic induction $(\mathrm{Bx})$; b) circuit for calculating the longitudinal component of the magnetic induction $(\mathrm{Bz})$; $\mathrm{c})$ circuit for calculating the total magnetic induction (B).

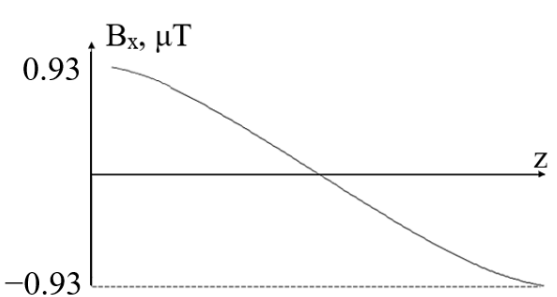

a

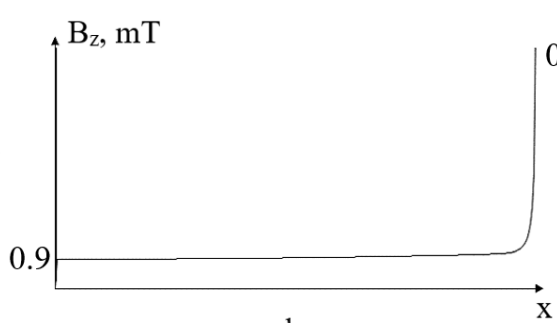

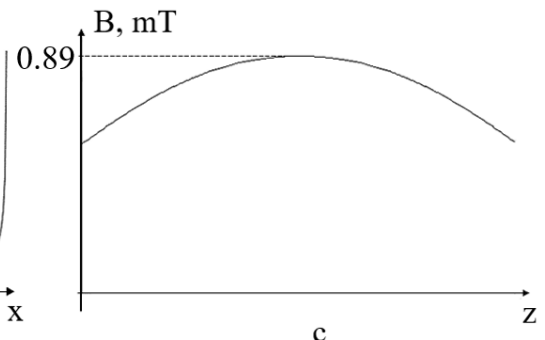

Fig. 3. Dependence graphs of the magnetic induction on the coordinate of the point: a) dependence of magnetic inductionx on the coordinate of the point on the longitudinal axis of the coil; b) the dependence of magnetic inductionz on the coordinate of the point along the radial axis of the coil; c) dependence of magnetic induction on the coordinate of a point along the central longitudinal axis.

$$
d B=\frac{\mu_{0}}{4 \pi} \times \frac{I d l \sin \alpha}{r^{2}}
$$

The current in the coil is assumed to be $1 \mathrm{~A}$. Because of computer simulation performed using the Pascal ABC programming language, we get the following dependencies (Fig. 3).

When the point moved along the $\mathrm{z}^{\prime}$ axis (Fig. 2a), parallel to the longitudinal axis of the device, the transverse component of the magnetic induction $\left(\mathrm{B}_{\mathrm{x}}\right)$ changed according to Fig. 3 a.

The Fig. 3a shows that the transverse component of the magnetic induction changes the direction of action when passing the geometric center of the device. However, its value in comparison with the longitudinal component is very small, and this component can be ignored in further calculations.

Then, a study was carried out of the dependence of the longitudinal component of the magnetic induction $(\mathrm{Bz})$ on the coordinate of the point along the radial axis of the coil $(\mathrm{Ox})$. When the point moved along the $\mathrm{x}$ axis (Fig.2b), the magnetic induction changed in accordance with the graph in Fig. 3b. In Fig. 3b shows that the magnetic induction along the transverse axis of the coil is distributed almost evenly. In the area located close to the walls of the device, the magnetic induction increases sharply.

However, since this effect acts mainly in the region of the walls of the device, and does not capture the emulsion itself, this increase can be ignored.

The distribution of the magnetic field inside the device is well illustrated in Fig. 3c. Dependence of the magnetic field induction on the coordinate along the longitudinal axis z shows in Fig. 2c. Let us compare the value of the induction at the geometric center of the coil (Fig.3c) with the value calculated theoretically (formula (2)):

$$
\begin{aligned}
& B_{\text {max }}=\frac{\mu \mu_{0} N I}{2 l}\left(\cos \alpha_{1}-\cos \alpha_{2}\right)= \\
& =\frac{4 \pi \cdot 10^{-7} \cdot 300 \cdot 1}{2 \cdot 0.3}\left(\cos 45^{0}-\cos 135^{\circ}\right)=0.89 \mathrm{mT},
\end{aligned}
$$

where $\alpha_{1}$ and $\alpha_{2}$ are the angles at which the edges of the coil are visible from its center.

Considering that $h=2 r$, we have, $\alpha_{1}=45^{\circ}$, $\alpha_{2}=135^{\circ}$ respectively. This ratio was used for computer modeling. Comparing the results of theoretical calculation and modeling, we are convinced of the correctness of the model. 
It is easy to determine the rms value of the magnetic induction at all points inside the coil according to the graph in Fig. 3c. This value will be approximately equal to the rms value of the magnetic induction at the points located on the $\mathrm{Oz}$ axis (formula (3)). The transverse component of the magnetic induction can be neglected according to the data in Fig. 3a. The value of induction also remains constant according to Fig. $3 \mathrm{~b}$ when distributed along the radial axis, $t$.

$$
B_{r m s}=\sqrt{\frac{1}{h} \int_{0}^{h} B^{2} d z}=0.785 \mathrm{mT} .
$$

The maximum induction value is determined by the current flowing through the turns of the coil according to formula (2). It is necessary to go in the calculation formulas from the maximum induction value to the rms value to determine the current and power consumed by the device. We introduce a coefficient that takes into account the inhomogeneous distribution of the magnetic field in the device:

$$
k=\frac{B_{r m s}}{B_{\text {max }}}=\frac{0.785}{0.89}=0.883 ;
$$

We optimize the parameters of the device in such a way that the power consumption tends to a minimum with a constant rms value of the magnetic induction. We derive the general formula for the dependence of the power consumption on the device parameters: $N$ is the number of turns, $d$ is the wire diameter, $h$ is the height of the cylinder and $r$ is its radius:

$$
P=\frac{8 B_{r m s}^{2} \rho}{\left(k \mu \mu_{0}\right)^{2}} \cdot \frac{\left(h^{2}+4 r^{2}\right) r}{N d^{2}}
$$

Consider that $h \approx 1.05 N d$, then:

$$
P=\frac{8.4 B_{r m s}^{2} \rho}{\left(k \mu \mu_{0}\right)^{2}} \cdot \frac{\left(h^{2}+4 r^{2}\right) r}{h d}
$$

Power consumption depends on the following factors and parameters:

1) the rms value of the magnetic induction, the distribution coefficient of the magnetic induction, the resistivity of copper and the magnetic permeability of the emulsion. These parameters cannot be changed in this expression.

2) the diameter of the turns. Power consumption is inversely proportional to the diameter of the turns.

$3)$ the geometric dimensions of the coil.

We determined the optimal parameters $h$ and $r$.

$$
h=\frac{V}{\pi r^{2}}
$$

Taking into account (5), we differentiate expression (4) with respect to $r$ and determine the optimal value of the device radius at which the consumption of active power from the network will be minimal:

$$
r=\sqrt[6]{\frac{V^{2}}{20 \pi^{2}}}
$$

With an installation volume of 5 liters (under laboratory conditions), the radius of the cylinder in which the effect of a constant magnetic field occurs will be:

$$
r=\sqrt[6]{\frac{0,005^{2}}{20 \pi^{2}}}=0.071 \mu
$$

Then the height of the cylinder will be, taking into account (5):

$$
h=r \sqrt{20}=0.071 \sqrt{20}=0.317 \mu .
$$

It can be seen from relation (6) that the device under development should have such a shape that its height exceeds the radius by about 4.47 times.

There are two main ways of connecting the unit to the mains supply: using a transformer and a controlled rectifier, or using an autotransformer and an uncontrolled rectifier. In the first case, the regulation of the rectified voltage value is carried out by changing the value of the firing angle of the thyristors, and in the second, by changing the number of turns of the secondary winding of the autotransformer.

We analyzed which of these methods would provide a more energy efficient process.

\subsection{Calculation of a circuit with a controlled rectifier and a transformer}

The most preferred single-phase rectifier circuit is an asymmetrical bridge circuit of a controlled rectifier, in which two transverse bridge arms are made on uncontrolled valves (diodes), and the other two - on controlled valves (thyristors). This circuit also has a higher power factor, better control response and a simpler rectifier thyristor control circuit (Fig. 4).

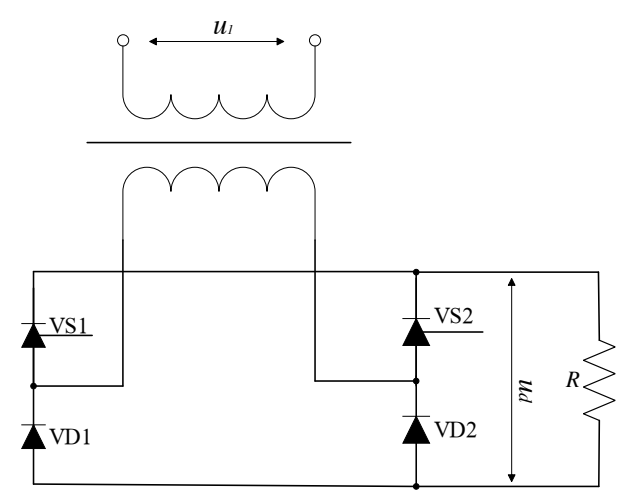

Fig. 4. Controlled rectifier circuit.

In fig. 4: $u_{1}$ is the effective value of the supply voltage, VD1 and VD2 are diodes, VS1 and VS2 are 
thyristors, $u_{d}$ is the value of the rectified voltage, $R$ is the load resistance, which is played by the coils of a constant magnetic field generator.

We have chosen the value of the control angle $\alpha=$ $25^{\circ}$, the short-circuit voltage $4.5 \%$ and calculate the main parameters of the power circuit for a 5-liter installation with different diameters of the winding wire.

The average voltage drop across the power diode is $1.5 \mathrm{~V}$, and across the thyristor $-20 \mathrm{~V}^{1}$. This is negatively affects the efficiency of the installation. The useful power is proportional to the voltage $U_{d}$. The current consumed by the device must remain constant according to the requirements of the technological process. An increase in energy efficiency implies an increase in the diameter of the winding wire. This leads to a decrease in resistance, and as a result, the voltage $U_{d}$ according to formula (4). Also this leads to an increase in power consumption from the network at low values of $U_{d}$. In addition, the efficiency of the installation also drops significantly. According to theoretical calculations, the consumed power $P_{l}$ will be minimal if the voltage drop in the rectifier is equal to the voltage drop at the terminals of the device to create a constant magnetic field, that is, with an efficiency of $50 \%$. This is confirmed by table 1 below. The data in tables 1,2 were obtained as a result of theoretical calculations based on Ohm's and Joule-Lenz's laws.

Table 1. Dependence of the efficiency and power of a 5L installation on the section of the winding wire.

\begin{tabular}{|r|r|r|r|r|}
\hline $\begin{array}{r}\text { Section } \\
s, \mathrm{~mm}^{2}\end{array}$ & $\begin{array}{c}\text { Voltage } \\
U_{d}, \mathrm{~V}\end{array}$ & $\begin{array}{c}\text { Net power } \\
P_{2}, \mathrm{~W}\end{array}$ & $\begin{array}{c}\text { Efficiency, } \\
\eta\end{array}$ & $\begin{array}{c}\text { Power } \\
\text { expended } \\
P_{1}, \mathrm{~W}\end{array}$ \\
\hline 0.5 & 236.71 & 9318.30 & 0.92 & 10164.66 \\
\hline 0.75 & 157.81 & 7608.65 & 0.88 & 8645.27 \\
\hline 1 & 118.36 & 6590.51 & 0.85 & 7787.71 \\
\hline 1.2 & 98.63 & 6017.08 & 0.82 & 7328.73 \\
\hline 1.5 & 78.90 & 5380.47 & 0.79 & 6846.56 \\
\hline 2 & 59.18 & 4659.15 & 0.73 & 6351.87 \\
\hline 2.5 & 47.34 & 4168.05 & 0.69 & 6060.92 \\
\hline 3 & 39.45 & 3805.50 & 0.65 & 5879.37 \\
\hline 4 & 29.59 & 3295.25 & 0.58 & 5689.66 \\
\hline $\mathbf{5}$ & $\mathbf{2 3 . 6 7}$ & $\mathbf{2 9 4 6 . 8 4}$ & $\mathbf{0 . 5 2}$ & $\mathbf{5 6 2 3 . 3 8}$ \\
\hline 6 & 19.73 & 2691.41 & 0.48 & 5624.87 \\
\hline 8 & 14.79 & 2329.57 & 0.41 & 5715.02 \\
\hline 10 & 11.84 & 2084.02 & 0.36 & 5869.77 \\
\hline 16 & 7.40 & 1647.63 & 0.26 & 6436.43 \\
\hline 25 & 4.73 & 1319.51 & 0.18 & 7311.92 \\
\hline 35 & 3.38 & 1115.50 & 0.14 & 8207.78 \\
\hline 50 & 2.37 & 932.30 & 0.10 & 9400.17 \\
\hline 70 & 1.69 & 789.17 & 0.07 & 10824.23 \\
\hline 95 & 1.25 & 676.39 & 0.05 & 12349.11 \\
\hline 120 & 0.99 & 602.41 & 0.04 & 13734.22 \\
\hline 150 & 0.79 & 538.75 & 0.04 & 15218.85 \\
\hline 185 & 0.64 & 485.60 & 0.03 & 16804.74 \\
\hline 240 & 0.49 & 426.02 & 0.02 & 18999.18 \\
\hline 300 & 0.39 & 380.78 & 0.02 & 21132.28 \\
\hline 400 & 0.30 & 330.47 & 0.01 & 24342.91 \\
\hline & & & & \\
\hline
\end{tabular}

The optimal section of the winding wire will be $5 \mathrm{~mm}^{2}$, while the power consumed from the network is minimal, and the voltage $U_{d}$ is as close as possible to $21.5 \mathrm{~V}$. The voltage drop in the rectifier branch.

\subsection{Calculation of a circuit with an uncontrolled rectifier and autotransformer}

The main advantage of such a circuit (Fig. 5) is the absence of thyristors, the voltage drop across which in the forward direction averages $20 \mathrm{~V}$. In fig. 5: $u_{l}$ is the effective value of the supply voltage, VD1 - VD4 is the diode bridge, $u_{d}$ is the value of the rectified voltage, $R$ is the load resistance, which is played by the coils of the constant magnetic field generator. We have updated Table 1 to take into account that thyristors are replaced by diodes in the uncontrolled rectifier circuit.

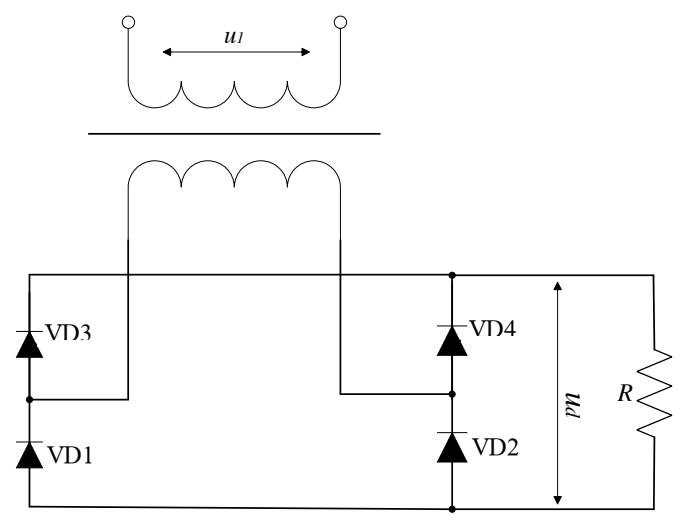

Fig. 5. Uncontrolled rectifier circuit uit.

In a circuit with an uncontrolled rectifier with an installation volume of 5 liters, with optimal overall dimensions and the correct choice of the winding crosssection, the power supplied to the rectifier should be about $2.1 \mathrm{~kW}$. Also note that the consumed power will be the smallest at an efficiency of $50 \%$, which is also confirmed by theoretical calculations. At the same time, in comparison with the power supply from a controlled rectifier, the installation becomes several times more efficient: for dehydration of the same volume of emulsion, the energy consumption will be 2.67 times lower. The design power of the autotransformer is only $2.5 \mathrm{~kW}$. If we build a similar table for an installation with a volume of 20 liters, we find that in this case the power consumption will be about $3.3 \mathrm{~kW}$, and for an installation with a volume of 100 liters $-5.7 \mathrm{~kW}$. Thus, the installation of an industrial design will have a much higher energy efficiency. 
Table 2. Dependence of the efficiency and device power on the section of the winding wire in a circuit with an uncontrolled rectifier (device volume is 5 liters).

\begin{tabular}{|r|r|r|r|r|}
\hline $\begin{array}{r}\text { Section } \\
s, \mathrm{~mm}^{2}\end{array}$ & $\begin{array}{c}\text { Voltage } \\
U_{d}, \mathrm{~V}\end{array}$ & $\begin{array}{c}\text { Net power } \\
P_{2}, \mathrm{~W}\end{array}$ & $\begin{array}{c}\text { Efficiency, } \\
\eta\end{array}$ & $\begin{array}{c}\text { Power } \\
\text { expended } \\
P_{l}, \mathrm{~W}\end{array}$ \\
\hline 0.5 & 236.71 & 9318.30 & 0.99 & 9436.40 \\
\hline 0.75 & 157.81 & 7608.65 & 0.98 & 7753.29 \\
\hline 1 & 118.36 & 6590.51 & 0.98 & 6757.56 \\
\hline 1.2 & 98.63 & 6017.08 & 0.97 & 6200.10 \\
\hline 1.5 & 78.90 & 5380.47 & 0.96 & 5585.04 \\
\hline 2 & 59.18 & 4659.15 & 0.95 & 4895.34 \\
\hline 2.5 & 47.34 & 4168.05 & 0.94 & 4432.17 \\
\hline 3 & 39.45 & 3805.50 & 0.93 & 4094.88 \\
\hline 4 & 29.59 & 3295.25 & 0.91 & 3629.36 \\
\hline 5 & 23.67 & 2946.84 & 0.89 & 3320.31 \\
\hline 6 & 19.73 & 2691.41 & 0.87 & 3100.73 \\
\hline 8 & 14.79 & 2329.57 & 0.83 & 2801.96 \\
\hline 10 & 11.84 & 2084.02 & 0.80 & 2612.27 \\
\hline 16 & 7.40 & 1647.63 & 0.71 & 2315.83 \\
\hline 25 & 4.73 & 1319.51 & 0.61 & 2155.66 \\
\hline $\mathbf{3 5}$ & $\mathbf{3 . 3 8}$ & $\mathbf{1 1 1 5 . 5 0}$ & $\mathbf{0 . 5 3}$ & $\mathbf{2 1 0 5 . 1 2}$ \\
\hline 50 & 2.37 & 932.30 & 0.44 & 2113.86 \\
\hline 70 & 1.69 & 789.17 & 0.36 & 2189.41 \\
\hline 95 & 1.25 & 676.39 & 0.29 & 2305.15 \\
\hline 120 & 0.99 & 602.41 & 0.25 & 2434.76 \\
\hline 150 & 0.79 & 538.75 & 0.21 & 2587.14 \\
\hline 185 & 0.64 & 485.60 & 0.18 & 2762.69 \\
\hline 240 & 0.49 & 426.02 & 0.14 & 3017.62 \\
\hline 300 & 0.39 & 380.78 & 0.12 & 3276.34 \\
\hline 400 & 0.30 & 330.47 & 0.09 & 3681.04 \\
\hline & & & & \\
\hline
\end{tabular}

\section{The discussion of the results}

During the research, we obtained the following results were obtained:

1) The distribution of a constant magnetic field inside the device under development has been determined.

2) The coefficient is determined, taking into account the inhomogeneous distribution of the magnetic field in the device: $k=0.883$.

3) The optimal geometric shape has been determined: $h=r \sqrt{ } 20$, where $h$ is the height of the cylinder in which the magnetic processing takes place, and $r$ is its radius.

4) The circuit with an autotransformer and an uncontrolled rectifier has the highest energy efficiency. So, for an installation with a volume of 5 liters, the energy consumption for this circuit will be 2.67 times lower than for a circuit with a transformer and a controlled rectifier.

5) The power consumed from the network was calculated for installations of various volumes. As a result, it was determined that energy efficiency increases significantly with increasing device performance. Thus, the production model of the device will have a much higher efficiency than the laboratory one.

\section{Conclusion}

In the course of the research, the results were obtained, which are necessary for the design of an experimental installation for dewatering an oil-water emulsion. The optimal geometric dimensions of the device and the optimal electrical power supply scheme were determined, and the power consumed from the network was calculated for installations of various volumes. As a result, it was determined that energy efficiency increases significantly with increasing device performance. These studies will make it possible to design a laboratory setup for carrying out a series of experiments on dehydration of an oil-water emulsion with a preliminary preparation stage in a constant magnetic field.

\section{References}

1. Z. Xi, L. Xiong, B. Gao, C. Cheng, Q. Lai, W. $\mathrm{He}$, The design and experimental research of high-power and high-frequency electromagnetic anti-scaling system, 2010 3rd International Conference on Biomedical Engineering and Informatics, Yantai, China, 1318-1321 (2010) DOI: 10.1109/BMEI.2010.5639257

2. A.N. Gulkov, A.N. Minaev, Magnetic field sea water treatment in ocean engineering systems, OCEANS 96 MTS/IEEE Conference Proceedings, The Coastal Ocean - Prospects for the 21st Century, Fort Lauderdale, FL, USA, 3, 1376-1377 (1996) DOI: 10.1109/OCEANS.1996.569104

3. A.I. Elshin, S.U. Ivlikov, The induction device for the electromagnetic processing of a liquid, Proceedings 6th Russian-Korean International Symposium on Science and Technology, KORUS-2002 (Cat. No.02EX565), Novosibirsk, Russia, 233-235 (2002) DOI: 10.1109/KORUS.2002.1028008

4. P.H.F. Silva, A.D.D. Neto, W. Damata, Electromagnetic field of an asymmetrically driven dipole antenna for heating dissipative media, MMET Conference Proceedings, 1998 International Conference on Mathematical Methods in Electromagnetic Theory, MMET 98 (Cat. No. 98EX114), Kharkov, Ukraine, 2, 559561 (1998) DOI: 10.1109/MMET.1998.709817

5. B.R. Rakhimov, Zh.U. Zhalolov, Application of magnetic fields in the process of desalination, Theory and practice of modern science, 249-251 (2019)

6. K.I. Khanifovich, M.D. Vilevich, Y.I. Flusovich, O.M. Valerievich, Magnetoelectrodehydrator, 2019 International Conference on Electrotechnical Complexes and Systems (ICOECS), Ufa, Russia, 1-5 (2019) DOI: 10.1109/ICOECS46375.2019.8950018

7. C. Li, W. Li, W. Li, C. Bin, Z. Zhao, Separation efficiency research on oil-water coalescence using Frequency pulse electromagnetic waves technology, 2011 Second International Conference on Mechanic Automation and Control Engineering, Inner Mongolia, China, 7664-7667 (2011) DOI: 10.1109/MACE.2011.5988825 
8. A.A. Voltsov, Intensification of the process of stratification of oil-water emulsions by means of their magnetic vibration treatment, Ufa, USPTU, 122 (2006)

9. A.M. Ermeev, A.A. Elpidinsky, On the use of a magnetic field in the processes of destruction of water-oil emulsions, Bulletin of Kazan Technological University, 16 (2), 170-174 (2013)

10. N.G. Ibragimov, A.N. Sudykin, R.Z. Sakhabutdinov, F.R. Gubaidulin, S.N. Sudykin, I.I. Urazov, Technologies and methods of intensification of the process of preparation of high-viscosity oil, Oil industry, 7, 61-63 (2016)

11. R. Zolfagari, A. Fakhru'l-Razi, L.S. Abdulla, Methods for demulsifying water-in-oil and oil-inwater emulsions in the petroleum industry, Sep. Purif. Technol., 170, 377-407 (2016)

12. A. Pedersen, E. Ildstad, A. Nysveen, Forces and movement of water droplets in oil caused by applied electric field," The 17th Annual Meeting of the IEEE Lasers and Electro-Optics Society, 2004. LEOS 2004, Boulder, CO, USA, 683-687 (2004) DOI: 10.1109/CEIDP.2004.1364342

13. C. Song, Q. Chen, X. Wang, L. Wen, T. Zheng, Deformation and motion behavior of droplets in a non-uniform electric field, 2015 IEEE 11th International Conference on the Properties and Applications of Dielectric Materials (ICPADM), Sydney, NSW, Australia, 652-655 (2015) DOI: 10.1109/ICPADM.2015.7295356

14. Z. Tian-yu, C. Qing-guo, L. Wen, S. Chun-hui, W. Xin-tao, Analysis of deformation and breakup of droplets in high voltage AC electric field, 2014 9th International Forum on Strategic Technology (IFOST), Cox's Bazar, Bangladesh, 268-271 (2014) DOI: 10.1109/IFOST.2014.6991119

15. P.R. Veles, N.A. Pivovarova, V.D. Shchugorev, V.M. Berdnikov, O.S. Shelamkova, I.N. Kulneva, A.T. Pivovarov, Method of dehydration of wateroil emulsion, Patent Russia 2152817 (20 July 2000)

16. P.R. Veles, N.A. Pivovarova, Magnetic tunnel, Patent Russia 2167824 (27 May 2001)

17. P.R. Veles, N.A. Pivovarova, V.M. Berdnikov, Magnetic activator for large flows of hydrocarbon raw materials, Utility model Russia, 38754 (10 July 2004)

18. G.V. Vlasova, E.V. Staroverova, N.A. Pivovarova, Influence of water and salt content in oil on the choice of dehydration process option, Oil and Gas Business, 1, 213-220 (2020)

19. A.D. Sheikh-Ali, Combined effect of magnetic field and demulsifiers on the process of oil dehydration, Oil and Gas Preparation, 1, 76-80 (2018)

20. M.H. Tavakoli, A. Ojagi, E. MohammadiManesh, M. Mansour, The influence of coil geometry on the process of induction heating in crystal growing systems, Journal of growing crystals, 6, 311 (2009)

21. K.K. Sum, Enhanced cavity filling passive power factor correction current driver, close to IEC/PCIM Magazine specification limits (February 1998)

22. S.G. Konesev, R.T. Khazieva, R.V. Kirillov, Inductive-capacitive converters for secondary high-voltage power supplies, International Multiconference on Industrial Engineering and Modern Technologies, Institute of Electrical and Electronic Engineers, FarEastCon, 1-5 (2019) DOI: 10.1109/FarEastCon.2019.8934018

23. S.G. Konesev, R.T. Khazieva, R.V. Kirillov, A.A. Konev, Investigation of the stabilizing properties of inductive-capacitive converters based on hybrid electromagnetic elements, Journal of Physics: Conference Series (JPCS), 803 (2017) DOI: 10.1088 / 1742-6596 / 803/1/012076

24. Y.M. Kashin, L.E. Kopelevich, V.A. Kim, Determination of the Parameters of the Electromagnetic System of the Installation for Oil Treatment, 2020 International Conference on Industrial Engineering, Applications and Manufacturing (ICIEAM), Sochi, Russia, 1-7 (2020)

DOI: 10.1109/ICIEAM48468.2020.9111990

25. M.D. Ivanov, S.G. Konesev, Generator of pulsed electromagnetic field for dehydration of oil-water emulsion, Materials of the 71st Scientific and Technical Conference of Students, Postgraduates and Young Scientists of USPTU, Ufa, USPTU, $572(2020)$ 